\title{
Are We Neglecting Nontuberculous Mycobacteria Just as Laboratory Contaminants? Time to Reevaluate Things
}

\author{
Pooja Sharma $\left(\mathbb{D},{ }^{1}\right.$ Digvijay Singh, ${ }^{2}$ Kusum Sharma, ${ }^{3}$ Santwana Verma, \\ Sanjay Mahajan, ${ }^{2}$ and Anil Kanga ${ }^{4}$ \\ ${ }^{1}$ Department of Microbiology, Dr Baba Saheb Ambedkar Medical College \& Hospital, Rohini, New Delhi 110085, India \\ ${ }^{2}$ Department of Microbiology, Indira Gandhi Medical College, Shimla, Himachal Pradesh 171001, India \\ ${ }^{3}$ Department of Microbiology, PGIMER, Chandigarh 160012, India \\ ${ }^{4}$ Department of Microbiology, Dr YSPGMC, Nahan, Sirmaur, Himachal Pradesh 173001, India
}

Correspondence should be addressed to Pooja Sharma; drpoojasharma86@gmail.com

Received 30 January 2018; Revised 15 April 2018; Accepted 10 May 2018; Published 21 June 2018

Academic Editor: Panagiotis Karanis

Copyright (C) 2018 Pooja Sharma et al. This is an open access article distributed under the Creative Commons Attribution License, which permits unrestricted use, distribution, and reproduction in any medium, provided the original work is properly cited.

\begin{abstract}
Objectives. Nontuberculous mycobacteria (NTM) incidences are on the rise worldwide, including the tuberculosis endemic areas. They should be identified rapidly to the species level and should be carefully differentiated as contamination, colonization, or disease. This study was aimed at determining the prevalence and clinicoepidemiological profile of mycobacteriosis cases. Materials and Methods. Cultures were made on liquid and solid media. NTM were identified by polymerase chain reaction (PCR) restriction analysis (PRA) and gene sequencing. Data was analyzed using Epi-info 7. Results. Out of the 1042 processed specimens, $16 \%$ were positive for M. tuberculosis complex and $1.2 \%$ for clinically significant NTM. M. intracellulare was the commonest species isolated. NTM were treated mainly on outdoor basis (92\%), involving more extrapulmonary system (62\%) and higher age-group of 41-60 years (69\%). No significant factor was seen to be associated clinically, radiologically, and biochemically with the NTM infections. Conclusions. Our study highlights the importance of early diagnosis and differentiation among Mycobacterium tuberculosis and NTM so that these NTM are not underestimated in routine diagnostic procedures merely as environmental or laboratory contaminants.
\end{abstract}

\section{Introduction}

Mycobacteria other than tuberculosis (MOTT) also known as nontuberculous mycobacteria (NTM) and atypical mycobacteria are organisms that are normally found in environmental soil and water [1]. They have been recognized as a cause of human disease since 1950s. At that time, NTMs isolation had been rare, and they were found almost exclusively in patients with underlying diseases [2]. Recent reports have shown that mycobacteriosis is on the rise even in healthy people. The distribution of NTM is nonuniform which appears to be geographically or environmentally dependent but still remains poorly defined. Geographic differences in distribution of NTM have been investigated comprehensively by Hoefsloot et al. in Europe, America, Australia, and few parts of Asian continent [3].
Disease caused by NTM is nonnotifiable. NTM are important pathogens because of their high level of antitubercular drug (ATT) resistance $[4,5]$. Recognizing the changing pattern of disease and epidemiology since 1980s with cases being reported in unrecognized populations, we initiated a study to assess the burden of such disease in our region $[6,7]$.

NTM are difficult to differentiate on the basis of clinical presentation from Mycobacterium tuberculosis complex (MTBC); thus it is necessary to identify NTM, as mere isolation especially from pulmonary sites does not establish the diagnosis. American Thoracic Society (ATS) guidelines are followed for the respiratory samples to correlate with clinical symptoms so that specific treatment can be instituted [5]. The aims of our study were (1) to determine isolation rate from pulmonary versus extrapulmonary sites, (2) to determine the patients' demographic characteristics and risk factors, (3) to 
determine effect of liquid versus solid culture on the isolation rate of NTM, and (4) to assess the disease incidence, the mycobacterial species involved, and the clinical conditions they caused.

\section{Material and Methods}

This prospective study was conducted in the tertiary care hospital at Shimla, Himachal Pradesh, India, over a period of one year from July 2013 to June 2014 on clinical samples from suspected patients of pulmonary and extrapulmonary mycobacterial infection.

2.1. Study Definitions. Differentiation between colonization and disease was done according to ATS 2007 guidelines in case of pulmonary specimens [5]. In extrapulmonary specimens, single isolation from sterile site as well as isolation for two or more times from nonsterile sites, along with the clinical correlation, was taken as confirmed disease [8].

2.2. Inclusion Criteria. Samples referred from patients admitted (IPD) or attending outdoor patient department (OPD) with clinical suspicion of pulmonary and extrapulmonary mycobacterial infection were included in the study.

2.3. Sample Collection and Transport. Sputum, bronchoalveolar lavage (BAL), body fluids, and aspirates (ascitic tap, pleural tap, synovial fluid, drains, pus discharge, gastric aspirates, and cerebrospinal fluid (CSF)) and early morning whole urine sample for three consecutive days collected in a sterile wide mouth screw capped container were received in the laboratory.

2.4. Sample Processing. Samples received were processed by digestion, decontamination, and concentration following the standard protocol [9]. Concentrated sample was divided into four parts.

(i) First part: This part was inoculated into MGIT 960 tube following manufacturer's instructions and tube was entered into the instrument [10]. From the positive tube, smear was prepared for Ziehl-Neelsen (ZN) and Gram's staining.

If positive for acid-fast bacilli (AFB), it was reported as instrument-positive and AFB-positive. It was then screened for production of MPT 64 antigen using Immunochromatographic Assay (TBcID) [11].

Those found positive for MPT 64 antigen were labeled as MTBC and those negative were labeled as NTM and were further identified by polymerase chain reaction (PCR) restriction analysis (PRA).

The protocol for PRA was the one described by Theresa B, Taylor and coworkers [12]. Briefly, hsp65 region was amplified using $\mathrm{Tb} 11$ and $\mathrm{Tb} 12$ primers. Restriction digestion of hsp65 PCR products was done with BstEII and
HaeIII enzymes. PRA algorithm was used to identify the isolates. Representative specimens were further confirmed by sequencing at PostGraduate Institute of Medical Education and Research, Chandigarh.

If microorganisms other than acid-fast bacilli were present, they were reported as instrumentpositive, AFB-negative, and contaminated.

If no microorganisms were present on the smear, the tube was reentered into the instrument. Negative cultures were removed as "out of protocol" negatives after 42 days.

(ii) Second part: This was inoculated on LowensteinJensen (LJ) medium and incubated at $37^{\circ} \mathrm{C}$. Bottles were examined daily for first 5-7 days and then weekly thereafter up to 8 weeks. If any growth was observed, a smear was made and heat fixed and $\mathrm{ZN}$ staining was done to confirm the AFB.

(iii) Third part: It was inoculated into the blood agar plates to rule out any contamination.

(iv) Fourth part: Smear was prepared, heat fixed, and stained by $\mathrm{ZN}$ method and examined under oil immersion.

2.5. Statistical Analysis. Data was entered on spreadsheet. Cross tabulation with outcome variable of interest was done using statistical software Epi-info version 7 (7.1.1.0).

\section{Result}

Out of the 1042 samples in the study, AFB were isolated from $18.6 \%(n=194)$ of samples. MTBC constituted $87 \%(168)$ and NTM were $13 \%$ (26) of total isolates.

Sensitivity of ZN, LJ, and MGIT-960 was 52\%, 17\%, and 97\% for NTM.

NTM isolated from pulmonary sites were M. intracellulare $62.5 \%$ (5), M. flavescens $12.5 \%$ (1), M. genavense $12.5 \%$ (1), and M. gordonae $12.5 \%$ (1).

Extrapulmonary NTM isolates included 6.5\% (5) M. intracellulare, $2.6 \%$ (2) M. abscessus, $1.3 \%$ (1) M. avium, $1.3 \%$ (1) M. mucogenicum, 1.3\% (1) M. austroafricanum, and $10.4 \%(8)$ M. gordonae.

Clinically significant NTM isolates were $50 \%$ (13) of the total NTM isolated in laboratory. They are summarized in Table 1. Among significant pulmonary isolates, 62.5\%(5), i.e., 4 sputum and 1 BAL cases, fulfilled the criteria. In extrapulmonary cases, isolates from synovial fluid (1), pleural fluid (2), and urine (5) were clinically significant. All the patients were nonreactive for human immunodeficiency virus (HIV).

NTM were treated mainly on outdoor basis $[92 \%$ (12)], involved extrapulmonary system more [62\% (8)], and affected higher age-group [41-60 years (69\%)]. History of tuberculosis was present in $31 \%$ (4) and radiological features of upper lung lobe involvement were seen in $60 \%$ of cases (Table 2).

NTM affected $41 \%$ (8) females and patients presented with fever and localized pain mainly [46\% (6)]. 54\% (7) 
TABLE 1: Clinical and microbiological features of clinically significant NTM.

\begin{tabular}{|c|c|c|c|c|c|c|c|}
\hline Spm no. & Isolate & Spm & $\begin{array}{c}\text { Chief } \\
\text { complaints }\end{array}$ & H/O ATT & Age & Sex & $\begin{array}{l}\text { No. of positive } \\
\text { sputum samples }\end{array}$ \\
\hline 1. & M. intracellulare & Sputum & $\begin{array}{l}\text { fever, productive } \\
\text { cough }\end{array}$ & 2 & 55 & M & 2 \\
\hline 2. & M. intracellulare & Sputum & haemoptysis & 2 & 22 & $\mathrm{~F}$ & 2 \\
\hline 3. & M. intracellulare & Sputum & haemoptysis & 1 & 49 & $\mathrm{~F}$ & 2 \\
\hline 4. & M. intracellulare & Sputum & $\begin{array}{l}\text { productive } \\
\text { cough, SOB }\end{array}$ & 3 & 40 & M & 3 \\
\hline 5. & M. gordonae & BAL & cough & 0 & 45 & $\mathrm{~F}$ & 1 \\
\hline 6. & M. intracellulare & Pleural Fluid & SOB & 0 & 60 & $\mathrm{~F}$ & 0 \\
\hline 7. & M. intracellulare & Pleural Fluid & $\begin{array}{l}\text { chest pain, } \\
\text { anorexia }\end{array}$ & 0 & 51 & $\mathrm{~F}$ & 0 \\
\hline 8. & M. intracellulare & Urine & $\begin{array}{l}\text { haematuria, } \\
\text { increased } \\
\text { frequency }\end{array}$ & 0 & 55 & M & NA \\
\hline 9. & M. abscessus & Urine & $\begin{array}{l}\text { sensation of } \\
\text { incomplete } \\
\text { evacuation }\end{array}$ & 0 & 45 & M & NA \\
\hline 10. & M. gordonae & Urine & $\begin{array}{c}\text { right } \\
\text { hypochondrium } \\
\text { pain, vague } \\
\text { lump in } \\
\text { abdomen } \\
\end{array}$ & 0 & 54 & $\mathrm{~F}$ & NA \\
\hline 11. & M. intracellulare & Urine & $\begin{array}{c}\text { increased } \\
\text { frequency of } \\
\text { urine }\end{array}$ & 0 & 37 & M & NA \\
\hline 12. & M. avium & Urine & $\begin{array}{c}\text { pain right } \\
\text { lumbar region }\end{array}$ & 0 & 27 & $\mathrm{~F}$ & NA \\
\hline 13. & M. abscessus & Synovial Fluid & $\begin{array}{l}\text { swelling right } \\
\text { ankle }\end{array}$ & 0 & 54 & $\mathrm{~F}$ & NA \\
\hline
\end{tabular}

NTM: nontuberculous mycobacteria; Spm: specimen; H/O ATT: history of antitubercular treatment; SOB: shortness of breath; NA: not applicable.

TABLE 2: Radiological features of patients with NTM isolates from sputum.

\begin{tabular}{lllc}
\hline S. No & Isolate & Spm & Radiological features \\
\hline 1 & M. intracellulare & Sputum & Patchy consolidation in right upper \& bilateral lower lobes \\
\hline 2 & M. intracellulare & Sputum & Cavitary lesion in right upper zone with in-homogeneous opacity in right middle zone \\
\hline 3 & M. intracellulare & Sputum & Patchy consolidation in right upper lobe. \\
\hline 5 & M. intracellulare & Sputum & Not available \\
\hline
\end{tabular}

NTM: nontuberculous mycobacteria; Spm: specimen; BAL: bronchoalveolar lavage.

of the patients were farmer by occupation. Erythrocyte sedimentation rate (ESR) was raised in 54\% (7), all had normal total leukocyte count (TLC), and one patient (8\%) had lymphocytosis.

\section{Discussion}

Mycobacteriosis being unnoticed in tuberculosis endemic areas is on the rise, being resistant to common antitubercular drugs [13]. Prevalence of clinically significant NTM among specimens received in our study was $1.2 \%$. Burden of mycobacteriosis is still vague due to underdiagnosis and lack of awareness. Developing countries lack facilities and expertise and often overlook these infections at peripheral levels.

We observed that extrapulmonary system was more involved with respect to pulmonary system with NTM, but other researchers mainly found the higher involvement of pulmonary system [14-17]. Age-group of patients most commonly affected by NTM was $41-60$ years (69\%) in our study. Other authors found the mean age to range between 43 and 59 years [15-17]. It has been postulated that immunosenescence 
leads to weaker immune response in elderly individuals resulting in increased susceptibility to infections, including mycobacteriosis $[18,19]$.

NTM being opportunistic pathogens have less tendency to cause infection. In a study by Dirac et al., immunosuppression and prior lung disease were associated risk factors for mycobacteriosis [20]. But all the patients in our study were HIV nonreactive. Commonest risk factor found in pulmonary mycobacteriosis cases in our study was preexisting comorbid pulmonary conditions like past history of tuberculosis. Tobacco or alcohol use was not found to be statistically associated with mycobacteriosis. High risk of NTM infections among patients with prior history of ATT intake was reported in earlier studies which support our present observation $[14,16]$. As stated earlier, mycobacteriosis among healthy individuals is not very rare [16]. Cases without recognized risk factors may have distinctive genetic susceptibility or environmental exposure to NTM [21]. Thus treating physician should keep mycobacteriosis in differential diagnosis in immunocompetent individuals also.

Pulmonary infections by NTM mostly involved the upper lobes of lungs as also observed by Costa et al. [22]. So it is difficult to differentiate this condition from tuberculosis which is also known to involve upper lobes more. Low hospitalization rate was seen among patients infected by NTM. This can be because of the mild pathogenic nature of NTM. Raised ESR though being very vague criterion in diagnosis of the NTM infection can be a contributory investigation. Neither increase nor decrease in blood cells count was seen with NTM infections. Thus, it is difficult to associate hematological laboratory parameters with NTM infection.

This study had few limitations. Firstly the number of patients with NTM isolates was small so as to conclude statistical differences. Secondly as this was tertiary care hospital based study, results might not represent the findings of population which could not attend this healthcare facility. Thus results may not be generalized to the community.

\section{Conclusion}

There is need for awareness regarding appropriate NTM diagnosis among physicians. NTM should be differentiated between colonization and disease. By using the rapid molecular methods, we can differentiate NTM from MTBC thus helping the clinician in early institution of specific treatment. PRA is cost-effective and can identify many mycobacterial species in a single experiment. These isolates are commonly disregarded as environmental contaminants in laboratories. One should be aware of increasing spectrum of these infections even in immunocompetent patients and more studies should be conducted to assess the importance and relevance of these isolates.

\section{Data Availability}

The data used to support the findings of this study are available from the corresponding author upon request.

\section{Conflicts of Interest}

The authors declare that they have no conflicts of interest.

\section{References}

[1] H. E. Crow, C. T. King, C. E. Smith, R. F. Corpe, and I. Stergus, "A limited clinical, pathologic, and epidemiologic study of patients with pulmonary lesions associated with atypical acid-fast bacilli in the sputum," American Review of Tuberculosis, vol. 75, pp. 199-222, 1957.

[2] A. Timpe and E. H. Runyon, "The relationship of "Atypical" acid-fast bacteria to human disease. A preliminary report," The Journal of Laboratory and Clinical Medicine, vol. 44, no. 2, pp. 202-209, 1954.

[3] W. Hoefsloot, J. Van Ingen, C. Andrejak et al., "The geographic diversity of nontuberculous mycobacteria isolated from pulmonary samples: an NTM-NET collaborative study," European Respiratory Journal, vol. 42, no. 6, pp. 1604-1613, 2013.

[4] D. Wagner and L. S. Young, "Nontuberculous mycobacterial infections: a clinical review," Infection, vol. 32 , no. 5, pp. 257270, 2004.

[5] D. E. Griffith, T. Aksamit, B. A. Brown-Elliott et al., "An official ATS/IDSA statement: diagnosis, treatment, and prevention of nontuberculous mycobacterial diseases," American Journal of Respiratory and Critical Care Medicine, vol. 175, no. 4, pp. 367416, 2007.

[6] J. O. Falkinham III, "Epidemiology of infection by nontuberculous mycobacteria," Clinical Microbiology Reviews, vol. 9, no. 2, pp. 177-215, 1996.

[7] E. Wolinsky, "Nontuberculous mycobacteria and associated diseases," American Review of Respiratory Disease, vol. 119, no. 1, pp. 107-159, 1979.

[8] Joint Tuberculosis Committee of the British Thoracic Society. Management of opportunistic mycobacterial infections: Joint Tuberculosis guidelines 1999. Thorax 2000; 55,210-18.

[9] P. T. Kent and G. P. Kubica, Public Health Mycobacteriology. A guide for the level III laboratory, US Department of Health and Human Services, CDC Atlanta, Georgia, 1985.

[10] BACTEC ${ }^{\mathrm{TM}}$ MGIT $^{\mathrm{TM}} 960$ System. Available from: URL: http:// www.bd.com/ds/technicalCenter/clsi/clsi-mgt960-7ml.pdf.

[11] BD MGIT ${ }^{\mathrm{TM}}$ TBc Identification Test. Available from: URL: http://www.bd.com/contentmanager/b_article.asp?Item_ID= 24199\&amp;ContentType_ID $=2 \&$ amp;BusinessCode $=20073 \&$ amp;d=home\&amp;s=uk\&amp;dTitle=BD+-+UK\%2FIreland $\&$ amp;dc=uk\&amp;dcTitle=BD+-+UK\%2FIreland.

[12] T. B. Taylor, C. Patterson, Y. Hale, and W. W. Safranek, "Routine use of PCR-restriction fragment length polymorphism analysis for identification of mycobacteria growing in liquid media," Journal of Clinical Microbiology, vol. 35, no. 1, pp. 79-85, 1997.

[13] V. M. Katoch, "Infections due to non-tuberculous mycobacteria (NTM)," Indian Journal of Medical Research, vol. 120, no. 4, pp. 290-304, 2004.

[14] S. Shenai, C. Rodrigues, and A. Mehta, "Time to identify and define non-tuberculous mycobacteria in a tuberculosisendemic region," The International Journal of Tuberculosis and Lung Disease, vol. 14, no. 8, pp. 1001-1008, 2010.

[15] E. Streit, J. Millet, and N. Rastogi, "Nontuberculous Mycobacteria in Guadeloupe, Martinique, and French Guiana from 1994 to 2012," Tuberculosis Research and Treatment, vol. 2013, pp. 1-8, 2013. 
[16] B. Varghese, Z. Memish, N. Abuljadayel, R. Al-Hakeem, F. Alrabiah, and S. A. Al-Hajoj, "Emergence of clinically relevant non-tuberculous mycobacterial infections in Saudi Arabia," PLOS Neglected Tropical Diseases, vol. 7, no. 5, Article ID e2234, 2013.

[17] D. P. O’Brien, B. J. Currie, and V. L. Krause, "Nontuberculous mycobacterial disease in northern Australia: a case series and review of the literature," Clinical Infectious Diseases, vol. 31, no. 4, pp. 958-967, 2000.

[18] P. J. Busse and S. K. Mathur, "Age-related changes in immune function: effect on airway inflammation," The Journal of Allergy and Clinical Immunology, vol. 126, no. 4, pp. 690-701, 2010.

[19] J. Ongrádi and V. Kövesdi, "Factors that may impact on immunosenescence: an appraisal," Immunity \& Ageing, vol. 7, article 7, 2010.

[20] M. A. Dirac, K. L. Horan, D. R. Doody et al., "Environment or host? A case-control study of risk factors for Mycobacterium avium complex lung disease," American Journal of Respiratory and Critical Care Medicine, vol. 186, no. 7, pp. 684-691, 2012.

[21] E. E. Bodle, J. A. Cunningham, P. Della-Latta, N. W. Schluger, and L. Saiman, "Epidemiology of nontuberculous mycobacteria in patients without HIV infection, New York City," Emerging Infectious Diseases, vol. 14, no. 3, pp. 390-396, 2008.

[22] A. R. F. da Costa, J. O. Falkinham III, M. L. Lopes et al., "Occurrence of nontuberculous mycobacterial pulmonary infection in an endemic area of tuberculosis," PLOS Neglected Tropical Diseases, vol. 7, no. 7, Article ID e2340, 2013. 


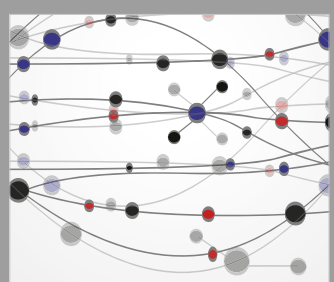

The Scientific World Journal
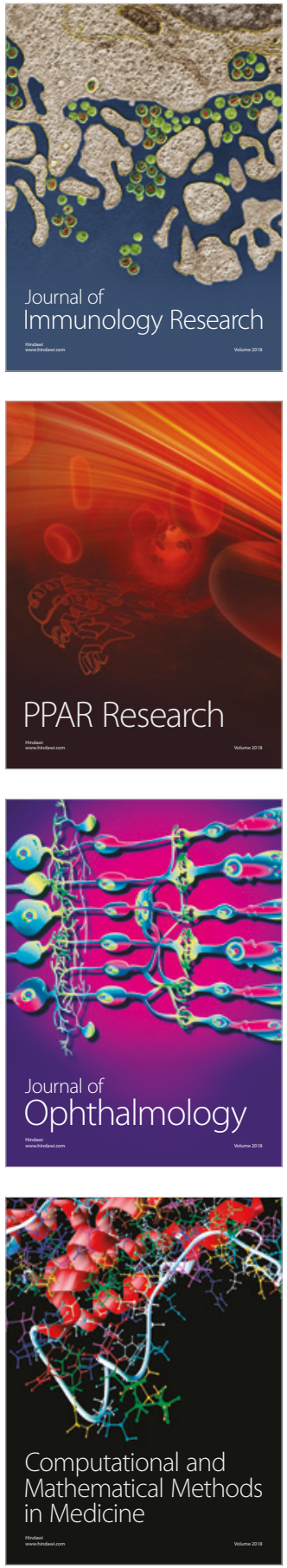

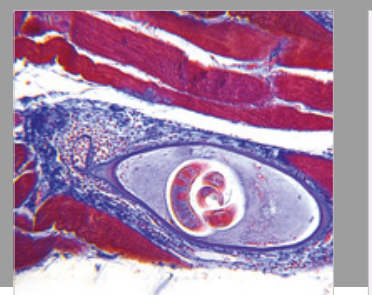

Gastroenterology Research and Practice

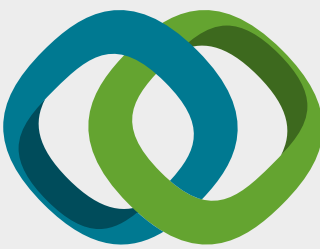

\section{Hindawi}

Submit your manuscripts at

www.hindawi.com
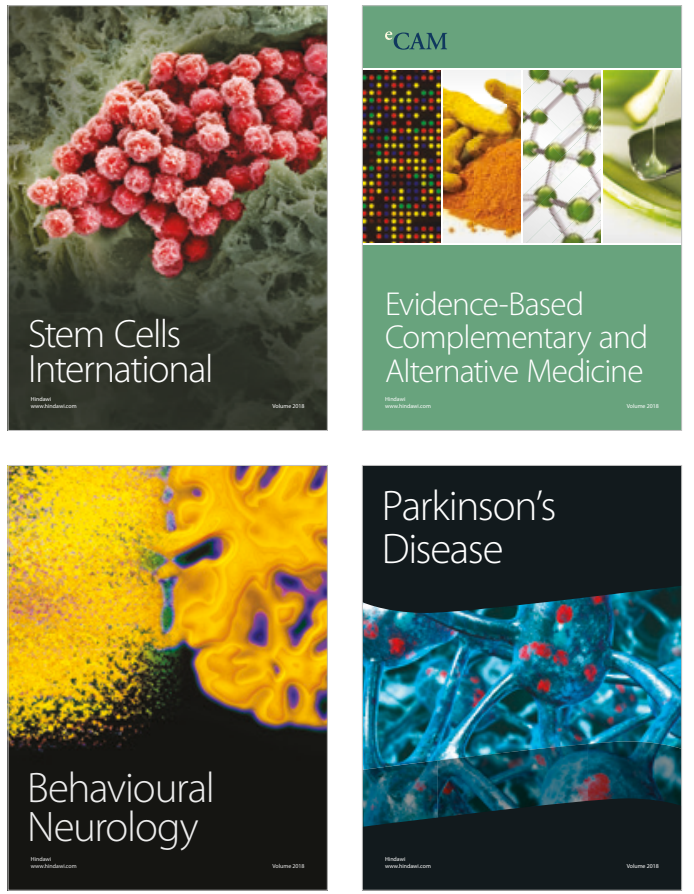

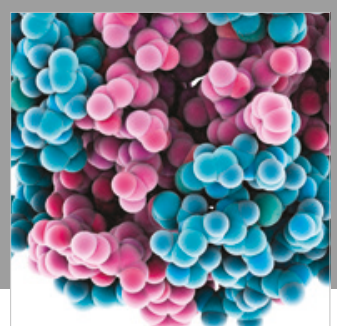

ournal of

Diabetes Research

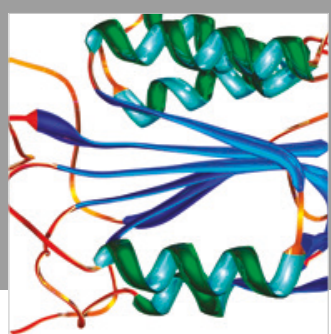

Disease Markers
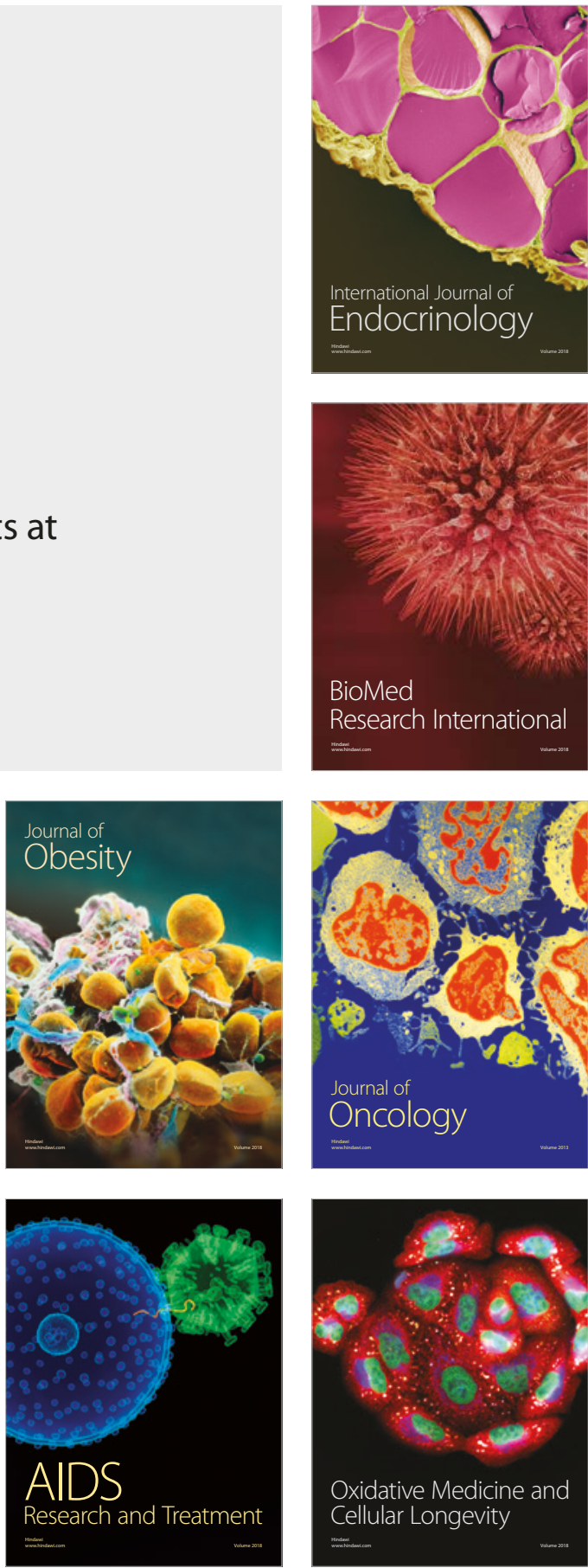